\title{
Fuzzy Control of Obstacle Avoidance for Mobile Robot Based on Single Chip
}

\section{Microcomputer}

\author{
WANG Ling \\ Baicheng Teachers College of Computer Science \\ Baicheng China \\ Century51@163.com
}

\begin{abstract}
This paper aimed at the environment is unknown, the mobile robot can replace or partially substitute for personnel to enter the scene implementation of environmental detection and upload information. A path planning method is proposed for obstacle avoidance of robot based on fuzzy control algorithm is given; and the membership functions of the fuzzy reasoning, and introduces fuzzy designer. System goals, a mobile robot using fuzzy control for path planning, based on the ultrasonic sensor to sense the surrounding environment, so as to reach the goal of autonomous robot.
\end{abstract}

Keywords_ path planning; fuzzy control; Atmega128;

\section{I .INTRODUCTION}

Mobile robot ${ }^{[1]}$ is able to perceive the external environment, dynamic planning routes, so as to realize the intelligent machine system of autonomous movement, the research involves a number of disciplines sensor technology, automatic control, artificial intelligence, computer vision, information technology. Real-time obstacle avoidance and navigation of mobile robot.

Mobile robot must be able to independently exercise must complete path planning, localization and obstacle avoidance path planning task, which has been the difficulty of the research of autonomous mobile robot, its task is to be from the robot to the initial state to the target state planning a collision free path, the robot can safe movement to the destination. Path planning of mobile robot can be divided into two types: global path planning and local path planning. Global path ${ }^{[2]}$ planning requires knowing the location information of all the roads on the path of robot walking in advance, and then according to these information using a certain method of planning a collision free path between the start point and end point of any given. Research on global path planning has been basically mature, the main methods of graph search method, the generalized cone method, the pose space law, vertex graph, regular grid method etc.. These methods in path planning requires a large amount of calculation in order to set up the environment model, the navigation process should continue to judge the robot and environment relative position, because of the uncertainty of environ ment information, sometimes leads to failu re, and the poor real-time performance. Local path planning without these defects, the utility model has the advantages of using local information, dynamic path planning, not only reduces the amount of calculation, and have a stronger ability to adapt to the environment, path planning out smooth and flat, real-time performance. The most typical representative of the local path planning is the artificial potential field method, its basic idea is that the motion of the robot in the environment as a virtual artificial stress field in the synthesis of movement, the robot and obstacles will exist between the draught force virtual virtual repulsion, and target, attraction and repulsion force of robot the acceleration or deceleration motion and change the direction of movement, until finally reached the destination.

Mobile robot obstacle ${ }^{[3]}$ avoidance and navigation has always been a pair of contradictions, navigation aims to enable the robot to the superior path from the start point to the end of movement, and the obstacle avoidance behavior in the process of movement and force the robot away from the end of the. Obstacle avoidance to the robot, the fundamental premise is found, so finding a more concise obstacles search algorithm, on the bas is of obstacle avoidance of robot, which is expected to solve the contradiction of obstacle avoidance and navigation. In addition, the robot is a highly nonlinear, strong coupling and time-varying system, more precise mathematical model is hard to establish the practical application, which makes the use of traditional control 
method is difficult. The fuzzy control method is mainly control experience of human, and on the basis of summarizing control ru les to achieve the control of robot. The obstacle avoidance and navigation of the fuzzy control method to realize the robot in recent years has made many achievements. Therefore, based on the main content of this paper is in search of the barricade, obstacle avoidance and navigation and obstacle avoidance fuzzy control method to realize the robot, which is simulated with visual $\mathrm{c}++$ programming, the effect is good. ${ }^{[4]}$

\section{FuZZY CONTROL}

Because fuzzy control does not need to establish a precise mathe matical model of the control system, but also in the process of designing the fuzzy controller, more adding control rules to human experience as the foundation, makes the design process is relatively simple, but the actual control effect is very good, in recent years has been rapid development.

\section{A. Fuzzy Membership Function}

In the fuzzy control for fuzzy reasoning are fuzzy variables, and between the position of the robot and the collision point to detect the actual distance $\mathrm{D}$, collision point azimuth angle $\alpha$ and deflection angle $\theta$ are accurate, so the fuzzy reasoning will be required before the exact amount of fuzzy. According to the distance to D size, the domain is divided into \{"near", "medium", "far" \}, i.e. $\{$ NEAR, MIDDLE, FAR $\}$. According to the azimuth of the size and in the navigation line on the range, the domain is divided into "negative", \{ negative", "negative", "zero", "s mall", " middle", "big" \}, i.e. \{NB, NM, NS, ZE, PS, PM, PB . Also the angle domain is divided into "negative", \{"negative", "negative", "zero", "s mall", "middle", "big" \}, i.e. \{NB, NM, NS, ZE, PS, PM, PB \}. According to the actual debug results, the distance of $0<\mathrm{d}<60$ is divided into NEAR, $20<\mathrm{d}<100$ is divided into MIDDLE, $d>60$ is divided into FAR, me mbership function shown in figure 1-1. The same azimuth $\alpha$, $90<\alpha<-60$ is divided into NB, $-60<\alpha<-60$ is divided into NS, $-40<\alpha<-60$ is divided into NM.

$-20<\alpha<20$ into ZE, $0<\alpha<40$ into PS, $20<\alpha<60$ into $\mathrm{PM}, 40<\alpha<90$ into $\mathrm{PB}$, me mbership function is shown in Figure 1 - 2. The fuzzy partition of deviation angle and azimuth angle, the membership function is shown in figure $1-3$.

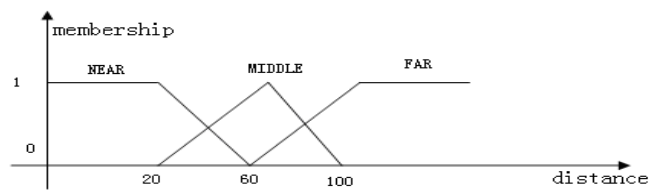

Figure 1 - 1 obstacle distance D me mbership function

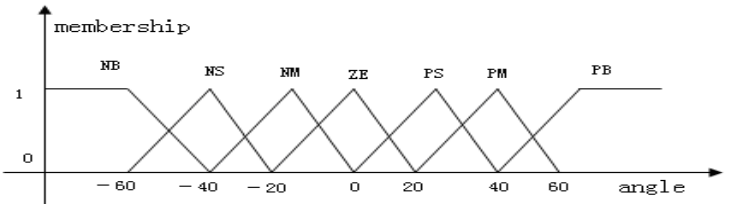

Figure 1 - 2 target azimuth $\alpha$ me mbership function

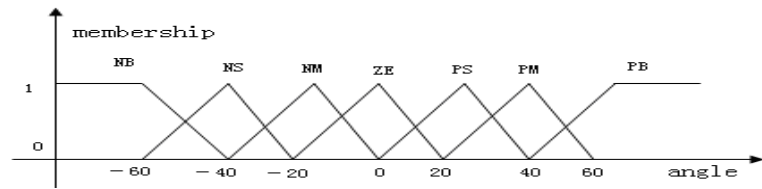

Figure 1-3 target deflection angle $\theta$ membership function

\section{B. Fuzzy Controller Design}

The basic principle of fuzzy control can be represented by Figure 1 - 4, the core part is a fuzzy controller, such as part of the map shown in the middle box. The control rules of fuzzy controller is realized by computer program ${ }^{[5-6]}$. Through the sampling is controlled by the precise value, then the value is compared to the error signal $\mathrm{E}$ with a given value. General error signal as the input of the fuzzy controller E. The input is an analog, after analog-dig ital conversion into digital quantity into fuzzy processing module and output fuzzy variable, the fuzzy quantities available corresponding fuzzy linguistic representation. We can get the fuzzy language error $\mathrm{E}$ is a subset of the set $\mathrm{E}$, e and fuzzy control rules (fuzzy R) make decisions based on compositional rule of inference, fuzzy control is obtained: $\mathrm{U}=\mathrm{e}^{\circ} \mathrm{R}$, where $\mathrm{u}$ is the fuzzy weight.

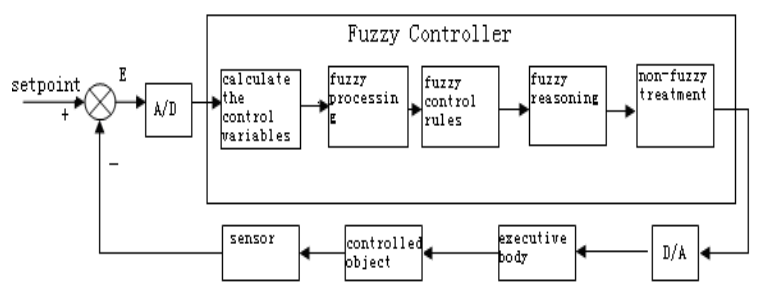

Figure 1-4 fuzzy controller 
In order to apply accurate control to the controlled object, there is also a need to fuzzy u into accurate value, this process is called non fuzzy processing (also known as fuzzy or clear processing). The precise digital control, the DAC is analog precision and then sent to the actuator, so as to realize the object control.

1) fuzzy control rules:A fuzzy control system is the key to the performance of the merits of the design of the fuzzy controller, usually have to design a fuzzy controller has the following steps.

a) the fuzzy fuzzy controller's input and output variables;

b) the control rules of fuzzy controller design, control experience in this process is actually the induction and the summary;

c) according to the method of actual control system selected fuzzy reasoning and defuzzification of the appropriate;

d) to determine the fuzzy controller's input and output variables of the domain, and to determine the parameters of the fuzzy controller.

The input of the fuzzy controller is the system deviation E, pass through fuzzy processing can use fuzzy linguistic variables to describe the deviation of $\mathrm{E}$, if $\mathrm{T}(\mathrm{E})$ E language value set, then:

$T(E)=\{N B, N M, N S, Z E, P S, P M, P B\}$.

If $T(U)$ that control the output of the $U$ language value set, then:

$T(U)=\{N B, N M, N S, Z E, P S, P M, P B\}$.

The fuzzy control rules can be expressed as:

RULE 1: IF E1 THEN U1 ELSE

RULE 2: IF E2 THEN U2ELSE

RULE 3: IF E3 THEN U3 ELSE

\section{RULE n: IF En THEN Un ELSE}

Each rule can construct a fuzzy relation $R i$, so the fuzzy rule for:

$$
R=R 1 \cap R 2 \cap R 3 \cap \ldots \cap R n \text {. If the known } E^{*},
$$

according to CRI synthesis reasoning method,

$$
U^{*}=E^{*} \circ R
$$

The membership function of R:

$$
u_{R}(x, y, z)=u_{A}(x) \wedge u_{B}(y) \wedge u_{C}(z)
$$

When the input variables to the fuzzy set $A, B, U$ output fuzzy variables can be expressed as:

$$
U=(A \times B) \circ R
$$

The membership function of $U$ :

$$
u_{U}(z)=u_{R}(x, y, z) \wedge\left(u_{A}(x) \wedge u_{B}(y), \forall x \in X,\right.
$$

$\forall y \in Y$

2) fuzzy reasoning algorithm:Fuzzy reasoning is based on solving the fuzzy output fuzzy quantity using the input fuzzy inference rules. Compared with the commonly used fuzzy inference rules for Mamdani maximum-minimum synthes is reasoning method of ${ }^{[7]}$.

$$
\text { LetA }^{i}, \mathrm{~B}^{i}, \mathrm{C}^{i} \text { on fuzzy language domain } \mathrm{X}, \mathrm{Y}, \mathrm{Z}
$$

on the set, the fuzzy relationship between each control rules for:

$$
\mathrm{R}^{i}=\left(\mathrm{A}^{i} \times \mathrm{B}^{i}\right) \times \mathrm{C}^{i} .
$$

The me mbership function of $\mathrm{R}^{i}$ for:

$$
u_{R i}(\mathrm{x}, \mathrm{y}, \mathrm{z})=u_{A i}(x) \wedge u_{B i}(y) \wedge u_{C_{i}}(z), \forall x \in X,
$$

$\forall y \in Y, \forall z \in Z$.

Set up a total of $\mathrm{N}$ fuzzy relation that is fuzzy

relation, total: $\mathrm{R}={ }^{\stackrel{n}{i=1}} \mathrm{R}^{i}$.

3) Defuzzification:The fuzzy output fuzzy inference obtained according to certain algorithm into a precise value to the control object, this process is called defuzzification, also became clear processing. There are 2 kinds of fuzzy method:

a) the maximum membership degree method of maximum membership degree method refers to the fuzzy inference to focus on selecting the maximum membership degree elements as the method to control the amount of. The method is simple and easy, but also eliminate the influence of other membership smaller element, poor control precision.

b) the method of center of gravity method considering all elements of control, high control precision. When the membership function of output is a 
continuous function, calculation formula of control volume can be expressed as:

$$
u^{*}=\frac{\int_{\min }^{\max } u \mu(u) d u}{\int_{\min }^{\max } \mu(u) d u}
$$

When the membership function of output as a discrete point set, calculation formula of control volume can be expressed as:

$$
u^{*}=\frac{\sum_{i=1}^{n}\left(u_{i} \mu_{i}\right)}{\sum_{i=1}^{n} \mu_{i}}
$$

Thus, after the above steps, the fuzzy controller has been designed.

\section{OBST ACLE AVOIDANCE, FUZZY CONTROL ALGORIT HM OF ${ }^{[8]}$}

As shown in Figure 2 - 1 shows $^{[9]}$, the first collision point $\mathrm{P}$ robot from a starting point to find along the navigation line direction, then according to the points and the current robot position (starting point) and the distance between the $\mathrm{D}$ distance and $\mathrm{P}$ azimuthal $\alpha$, co mbining the fuzzy control algorith $m$ to make the robot to offset to a point of $\theta$ can successfully avoid moving collision point $\mathrm{P}$ and to the $\mathrm{P}^{\prime}$ position. The point $\mathrm{P}^{\prime}$ is the origin, as a new $\mathrm{P}^{\prime}$ and the end of the line, and this line as the axis $X^{\prime}$, perpendicular to the $X^{\prime}$ axis on the $Y^{\prime}$ axis is the establishment of a new coordinate system, the coordinate system according to the obstacle search algorith $m$ to find the next collision point, once again the implementation of fu zzy control algorithm. Because the robot in the process of moving always along the real-time navigation line direction, in theory should be able to successfully reach the end of the final ${ }^{[10]}$.

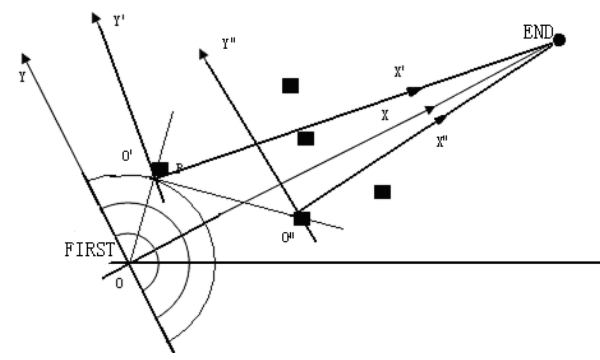

Figure $2-1$ Schematic diagram for the search of obstacles
As shown in Figure 2 - 1 shows coordinates, set the current robot position coordinates $\left(\mathrm{x}_{0}, \mathrm{y}_{0}\right)$, collision point $P$ coordinates to $(x, y)$, end coordinates $\left(x_{d}, y_{d}\right)$ for calculation of $\mathrm{D}$, distance and azimuth $\alpha$ by:

$$
d=\sqrt{\left(x-x_{0}\right)^{2}+\left(y-y_{0}\right)^{2}}
$$

$\alpha=a \tan \left(\left(y-y_{0}\right) /\left(x-x_{0}\right)\right)-a \tan \left(\left(y_{d}-y_{0}\right) /\left(x_{d}-x_{0}\right)\right)$

If during the movement of the robot, bypassing the mobile collision point $\mathrm{P}$ and $\mathrm{P}^{\prime} \quad\left(\mathrm{x}^{\prime}, \mathrm{y}^{\prime}\right)$, then the robot's current coordinate is set to $\left(\mathrm{x}^{\prime}, \mathrm{y}^{\prime}\right)$, the coordinate values into the formula to calculate the distance of $\mathrm{D}$ and azimuth angle $\alpha$, so the cycle until the robot reaches the end calculation.

The above process is assumed to search to search radius collision point when $\mathrm{P}$ is $\mathrm{R}$, the azimuth angle $\alpha$, according to the fuzzy control rules for the deflection angle $\theta$.

\section{OBST ACLE AVOIDANCEPATHS PLANNING FOR} FUZZY CONTROL RULES

This process will distance D fuzzy: NEAR, MIDDLE, FAR three grades, azimuth a mbiguity: NB, NM, NS, ZE, PS, PM, PB seven grades, according to people driving experience all can get 21 control rules, the fuzzy control rules using "IF THEN" the statement as follows:

RULE 1: If $\mathrm{d}$ IS FAR AND $\alpha$ IS PS THEN $\theta$ IS NS;

RULE 2: If $d$ IS FAR AND $\alpha$ IS PM

THENO IS NM;

RULE 3: If $\mathrm{d}$ IS FAR AND $\alpha$ IS PB THENO IS NB;

RULE 4: If $\mathrm{d}$ IS FAR AND $\alpha$ IS ZE THENO IS NS ORO IS PS ;

RULE 11: If $\mathrm{d}$ IS MIDDLE AND $\alpha$ IS ZE THENO IS NM OR $\theta$ IS PM;

RULE 18: If $d$ IS NEAR AND $\alpha$ IS ZE THENO IS NB OR $\theta$ IS PB;

The rule 4, 11, 18 shows whether the current robot position and the obstacle distance regardless of distance, as long as the azimuthal $\alpha=0$, namely the obstacles in the 
connection position of the mobile robot and the end, the deflection angle of $\theta$ can be positive or negative; rule $15 \sim 21$ shows when the obstacle is near robot, deflection angle $\theta$ must be large enough to avoid obstacles. Thus it can be seen, these rules directly from people's driving experience.

\section{SIMULATION RESULTS}

In order to verify the feasibility and effectiveness of the presented algorithm, the simulation experiments, simulation results show that the algorithm has good feasibility.

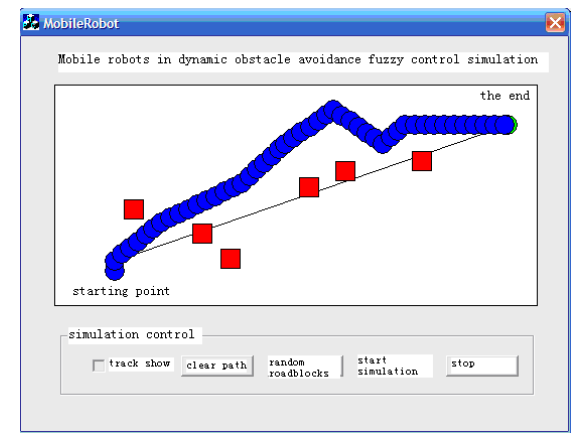

Figure 4 - 1 obstacle avoidance simulation results

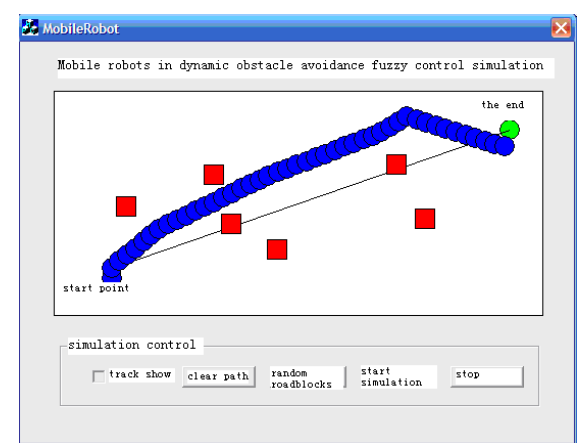

Figure 4 - 2 obstacle avoidance simulation results

\section{CONCLUSION}

This paper discusses the design scheme of robot path control based on ATmage128 single chip microcomputer, walking robot with obstacle avoidance path planning based on fuzzy control, infrared sensors to sense the surrounding environment, thereby robot reaches the preset target autonomous mobile. The paper gives the membership function of the fuzzy control, obstacle avoidance, fuzzy control rules is introduced.

\section{Reference}

[1] Cai Zixing. Beijing: Tsinghua University press, 2000, 2-3, 6-7
[2] Sebastian Thrun, Dieter Fox, Wolfram Burgard. Probabilistic Robotics. Cambridge, MA: The MIT Press. 2005

[3] Xu Yuanchang. Industrial robot. Beijing: China Light Industry Press, $1998,9-11$

[4] Sun Fuchun. Introduction to robotics. Beijing: Electronic Industry Press, 2002, 12-13

[5] Zhao Xianzhang. A path planning method for mobile robot based on particle swarm optimization algorithm, computer application, 2007

[6] Liu Jingjing. And its application in robot path planning in the multi-objective genetic optimization (Master Thesis). Nanjing: Nanjing University of Science and Technology, 2007

[7] Wang Shuai. Reinforcement learning algorithm for mobile robot path planning, underground electrical technology based on [8], 2008 Huang Bingqiang robot, path planning and control technology based on fuzzy controller, 2007

[8] Zhang Yan. The complete RPC algorithm for robot path planning in unknown environment, computer engineering and applications, 2007

[9] Cody Kwork, Dieter Fox, Marina Meila. Adaptive Real-timeParticle Filters for Robot Localization. Proc. of the IEEE International Conference on Robotics and Aut omation (ICRA), 2003

[10] Simon Godsill, Tim Clapp. Improvement strategies for Monte Carlo particle filters [D].Signal Processing Group, University of Cambridge, 1998 